\title{
ARTICLE \\ ZYZ-803, a novel hydrogen sulfide-nitric oxide conjugated donor, promotes angiogenesis via cross-talk between STAT3 and CaMKII
}

\author{
Ying Xiong ${ }^{1}$, Ling-ling Chang ${ }^{1}$, Bahieu Tran ${ }^{1}$, Tao Dai ${ }^{1}$, Rui Zhong ${ }^{1}$, Yi-cheng Mao ${ }^{1}$ and Yi-zhun Zhu ${ }^{1,2}$
}

\begin{abstract}
Endothelial angiogenesis plays a vital role in recovery from chronic ischemic injuries. ZYZ-803 is a hybrid donor of hydrogen sulfide $\left(\mathrm{H}_{2} \mathrm{~S}\right)$ and nitric oxide (NO). Previous studies showed that ZYZ-803 stimulated endothelial cell angiogenesis both in vitro and in vivo. In this study, we investigated whether the signal transducer and activator of transcription 3 (STAT3) and Ca ${ }^{2+} / \mathrm{CaM}$-dependent protein kinase II (CaMKII) signaling was involved in ZYZ-803-induced angiogenesis. Treatment with ZYZ-803 (1 $\mu$ M) significantly increased the phosphorylation of STAT3 (Tyr705) and CaMKII (Thr286) in human umbilical vein endothelial cells (HUVECs), these two effects had a similar time course. Pretreatment with WP1066 (STAT3 inhibitor) or KN93 (CAMKII inhibitor) blocked ZYZ-803-induced STAT3/CAMKII activation and significantly suppressed the proliferation and migration of HUVECs. In addition, pretreatment with the inhibitors significantly decreased ZYZ-803-induced tube formations along with the outgrowths of branch-like microvessels in aortic rings. In the mice with femoral artery ligation, administration of ZYZ-803 significantly increased the blood perfusion and vascular density in the hind limb, whereas co-administration of WP1066 or KN93 abrogated ZYZ-803-induced angiogenesis. By using STAT3 siRNA, we further explored the cross-talk between STAT3 and CaMKII in ZYZ-803-induced angiogenesis. We found that STAT3 knockdown suppressed ZYZ-803-induced HUVEC angiogenesis and affected CaMKII expression. ZYZ-803 treatment markedly enhanced the interaction between CaMKII and STAT3. ZYZ-803 treatment induced the nuclear translocation of STAT3. We demonstrated that both STAT3 and CaMKII functioned as positive regulators in ZYZ-803-induced endothelial angiogenesis and STAT3 was important in ZYZ-803-induced CaMKII activation, which highlights the beneficial role of ZYZ-803 in STAT3/CaMKII-related cardiovascular diseases.
\end{abstract}

Keywords: ZYZ-803; $\mathrm{H}_{2} \mathrm{~S}$; NO; human umbilical vein endothelial cells (HUVECs); angiogenesis; STAT3; CaMKII

Acta Pharmacologica Sinica (2020) 41:218-228; https://doi.org/10.1038/s41401-019-0255-3

\section{INTRODUCTION}

Angiogenesis, the formation of new capillaries from preexisting blood vessels, is a complex multistage process involving endothelial cell proliferation, selective surrounding extracellular matrix degradation, endothelial cell migration and tubular structure formation. Not surprisingly, endothelial angiogenesis plays a vital role in recovery from chronic and ischemic injuries [1].

ZYZ-803, a novel hybrid donor of hydrogen sulfide $\left(\mathrm{H}_{2} \mathrm{~S}\right)$ and nitric oxide (NO) developed by our lab, exerts a powerful protective effect on the cardiovascular system. As reported, ZYZ803 regulated vascular tone in isolated rat aortic rings [2], attenuated cardiac dysfunction and improved myocardial injury after heart failure [3]. In addition, ZYZ-803 significantly stimulated endothelial cell angiogenesis both in vitro and in vivo [4].

Accumulating evidence from pharmacologic studies suggests that both $\mathrm{H}_{2} \mathrm{~S}$ and $\mathrm{NO}$ serve as potent angiogenic molecules acting individually or in combination with other angiogenic factors to promote endothelial cell proliferation, migration and tube formation [5-11]. As a novel $\mathrm{H}_{2} \mathrm{~S}-\mathrm{NO}$-releasing molecule, ZYZ-803 was developed by coupling S-propargyl-cysteine (SPRC) with furoxan; however, ZYZ-803 demonstrated significantly slower release of $\mathrm{H}_{2} \mathrm{~S}$ and $\mathrm{NO}$ than SPRC and/or furoxan. In our previous study, $\mathrm{H}_{2} \mathrm{~S}$ and NO from $\mathrm{ZYZ}-803$ promoted angiogenesis through the SIRT1/VEGF/cGMP pathway [4].

In addition to SIRT1, which serves as a potential therapeutic cardiovascular target related to $\mathrm{H}_{2} \mathrm{~S}-\mathrm{NO}$, there are still many other angiogenic factors. Signal transducer and activator of transcription 3 (STAT3), an important member of the STAT protein family, plays a crucial role in the regulation of angiogenesis. It has been shown that SPRC, as a water-soluble modulator of endogenous $\mathrm{H}_{2} \mathrm{~S}$, can trigger angiogenesis via a STAT3/VEGFR2-mediated mechanism [12]. $\mathrm{Ca}^{2+} / \mathrm{CaM}$-dependent protein kinase II (CaMKII), a serine/ threonine-specific protein kinase regulated by the $\mathrm{Ca}^{2+} /$ calmodulin complex, has also received more attention for its role in the cardiovascular system. The $\mathrm{PLC} / \mathrm{IP} 3 / \mathrm{Ca}^{2+} / \mathrm{CaMKIl}$ signaling pathway was reported to be involved in VEGF-induced retinal angiogenesis [13]. Furthermore, several studies have proven that the eNOSCaMKII axis is associated directly with angiogenesis [14-16].

More interestingly, accumulating evidence reveals that there is cross-talk between STAT3 and CAMKII. For example, IL- 6 activated

${ }^{1}$ Institute of Biomedical Science and School of Pharmacy, Fudan University, Shanghai 200032, China and ${ }^{2}$ School of Pharmacy, Macau University of Science and Technology, Macau, China

Correspondence: Yi-cheng Mao (maoyc@fudan.edu.cn) or Yi-zhun Zhu (yzzhu@must.edu.mo)

Received: 8 December 2018 Accepted: 21 May 2019

Published online: 17 July 2019 
STAT3 via a CaMKII-dependent manner in pressure overloadinduced left ventricular hypertrophy and dysfunction [17]. CaMKIly enhanced epithelial STAT3 activation to promote the survival and proliferation of colonic epithelial cells during colitis-associated colorectal cancer development [18]. Moreover, CaMKIly directly phosphorylates and activates STAT3 at Ser727 both in vitro and in vivo, which indicates that STAT3 is likely to be a direct substrate of CaMKIly in myeloid leukemia cells [19].

However, whether STAT3/CaMKII is involved in the process of ZYZ-803-induced angiogenesis has not yet been reported. Based on a previous study of $\mathrm{H}_{2} \mathrm{~S}-\mathrm{STAT3}$ and the NO-CaMKII axis, we thought it would be interesting to use ZYZ-803 as a dual-gas transmitter modulator of both $\mathrm{H}_{2} \mathrm{~S}$ and $\mathrm{NO}$ to learn about the cross-talk between the two axes. Thus, in the current study, ZYZ803 was used to investigate the molecular mechanisms of STAT3 as well as CAMKII in mediating $\mathrm{H}_{2} \mathrm{~S}$-NO-induced angiogenesis in human umbilical vein endothelial cells (HUVECs).

\section{MATERIALS AND METHODS}

Drugs and solutions

ZYZ-803 was synthesized by the reaction of 2-amino-3-propynylsulfanyl-propionic acid with cinnamyl alcohol and purified as described before [2]. WP1066 and KN93 were purchased from Medchemexpress LLC (Monmouth Junction, NJ, USA). PAG and LNAME were purchased from Sigma-Aldrich (St. Louis, MO, USA). The primary antibodies used were as follows: anti-STAT3 and antiGAPDH were purchased from Proteintech (Wuhan, Hubei, China); anti-p-STAT3, anti-p-CAMKII and anti-CAMKII (pan) were purchased from Cell Signaling Technology (Beverly, MA, USA); antiPCNA and anti-LaminB1 were from Santa Cruz Biotechnology (Santa Cruz, CA, USA); and anti-cyclin D1 was from Bioss (Beijing, China). Horseradish peroxidase (HRP)-conjugated secondary antibodies were from Jackson Laboratories (West Grove, PA, USA). The EdU Assay Kit was purchased from Beyotime Institute of Biotechnology (Shanghai, China).

\section{Animals}

Male C57BL/6 mice ( 8 weeks old) and Sprague Dawley (SD) rats (6-7 weeks old) were maintained under standard conditions and with free access to food and water. All animals were housed according to the "Guide for the Care and Use of Laboratory Animals" published by the National Institutes of Health (NIH). All experimental procedures were performed according to the local ethical committee of Fudan University. All animals were purchased from the Animal Center of Slaccas (Shanghai, China).

\section{Cell culture}

HUVECs were purchased from the American Type Culture Collection (ATCC, San Francisco, CA, USA) and cultured in Dulbecco's modified Eagle's medium (HyClone) with $10 \%$ fetal bovine serum (Atlanta Biologicals, Flowery Branch, GA, USA) in 5\% $\mathrm{CO}_{2}$ saturated with $\mathrm{H}_{2} \mathrm{O}$.

\section{Proliferation assay}

HUVECs were plated in 24-well plates and treated with ZYZ-803 in the presence or absence of inhibitors or siRNA. After $24 \mathrm{~h}$ of incubation, cell proliferation activity was assessed using a commercially available EdU Assay Kit (Beyotime Biotechnology, China) according to the protocol provided. The nuclei of proliferated cells were dyed green, while the nuclei of all cells were dyed blue with DAPI (4', 6-diamidino-2-phenylindole).

Transwell migration assay

HUVECs were cultured in the upper plate compartment $(8-\mu \mathrm{m}$, 24-well insert, Millipore, USA), while the drug was added to the lower plate compartment in the presence or absence of inhibitors or siRNA. After incubation at $37^{\circ} \mathrm{C}$ for $24 \mathrm{~h}$, the migrated cells on the bottom surface of the membrane were fixed and stained with $0.1 \%$ crystal violet, while the non-migratory cells in the upper compartment were removed with a cotton swab. Cells in three random fields from each well were counted to determine the average cell number.

Wound-healing assay

HUVECs were plated at confluence on 12-well plates. Then, the cells were scraped with a sterile $200-\mu \mathrm{L}$ pipette tip to generate a scratch wound and gently washed 3 times with PBS to remove the detached cells. The drug was added to the wells in the presence or absence of inhibitors or siRNA. Images were taken at 0 and $24 \mathrm{~h}$ with a Zeiss digital camera (Zeiss, Oberkochen, Germany). Wound areas were analyzed using ImageJ software (NIH, USA).

\section{Tubulogenesis assay}

HUVECs were pretreated with test compounds for the indicated time and then seeded on 48-well plates, which were precoated with $150 \mu \mathrm{L}$ of Matrigel (Growth Factor Reduced, BD Biosciences). Tubular structures were photographed after $6 \mathrm{~h}$ of incubation with ZYZ-803 and quantified by measuring the length of each tube using ImageJ software. The tube length in three random fields from each well was calculated to determine the average length.

\section{Rat aortic ring assay}

Aortic ring assays were performed as described previously [12]. In brief, a 96-well plate was coated with $50 \mu \mathrm{L}$ of Matrigel (BD Biosciences). SD rats were anesthetized intraperitoneally with $10 \%$ chloral hydrate and dissected to obtain isolated aortae. The thoracic aorta was cut into small ring-like segments, laid on the top of Matrigel and covered with another $100 \mu \mathrm{L}$ of Matrigel. After solidification, the aortic rings were cultured in DMEM with $10 \%$ FBS. The drug was added to the wells in the presence or absence of inhibitors, and the medium and the drug were changed every 2 days. After 6 days of culture, the aortic rings were photographed, and the areas of microvessels were calculated and statistically analyzed.

\section{Murine hind limb ischemia (HLI) model}

The methodology and surgical tools for creating and evaluating the murine $\mathrm{HLI}$ model were used as described in the literature [20]. Briefly, $\mathrm{HLI}$ was surgically induced via femoral artery ligation in 8-week-old C57BL/6J mice. Then, the $\mathrm{C} 57 \mathrm{BL} / 6 \mathrm{~J}$ mice were injected intraperitoneally with ZYZ-803 $(8.7 \mathrm{mg} / \mathrm{kg}$ per day), WP1066 $(5.0 \mathrm{mg} / \mathrm{kg}$ per day) or KN93 $(5.0 \mathrm{mg} / \mathrm{kg}$ per day) the day after HLI induction. A MoorLDI2-2 laser Doppler imaging system (Moor Instruments, Devon, UK) was used to follow the changes in blood flow recovery over time.

Immunohistochemistry

Fourteen days after $\mathrm{HLI}$, the mice were sacrificed, and their gastrocnemius muscles were fixed in paraformaldehyde immediately after excision from the operative hind limbs and then embedded in paraffin. The endothelial cells in the tissue were stained with a rabbit polyclonal CD31 antibody (Abcam, Cambridge, UK). Five random fields from each section were imaged with a Zeiss digital camera. Vascular density was quantified with Image-Pro Plus 6 software.

\section{RNA interference}

HUVECs were seeded in 6-well plates at $30 \%-50 \%$ confluence the day before transfection. Lipofectamine RNAiMax (Thermo Fisher Scientific, Shanghai, China) was used to transfect $50 \mathrm{nM}$ STAT3 siRNA (sc-29493, Santa Cruz Biotechnology, CA, USA) into HUVECs in OPTI-MEM I Reduced Serum Medium (Gibco) for $48 \mathrm{~h}$. The medium was changed $7 \mathrm{~h}$ after transfection. 
Real-time quantitative RT-PCR analysis

Total RNA from the HUVECs was isolated using TRlzol reagent. cDNA was prepared with a PrimeScript 1st Strand cDNA Synthesis Kit (TaKaRa) and then amplified by PCR with specific primers. Relative gene expression was determined using a BIO-RAD IQ5 system. Primer sequences used for the PCR assay were as follows: GAPDH forward, 5'-GATTCCACCCATGGCAAATTCC-3', and reverse, 5'GCATCGCCCCACTTGATTTI-3'; STAT3 forward, 5'-AACAGGATGGCC CAATGGAA-3', and reverse, 5'-AAGCGGCTATACTGCTGGTC-3'.

Protein extraction and nuclear isolation

Nuclear and cytoplasmic proteins were collected using nuclear and cytoplasmic extraction reagents (G-Biosciences) according to the manufacturer's instructions.

\section{Western blotting}

Protein extracts prepared from HUVECs were separated on sodium dodecyl sulfate-polyacrylamide gels and subsequently transferred onto nitrocellulose membranes. After being blocked with $10 \%$ nonfat milk in Tris- $\mathrm{HCl}$ buffered saline, the membranes were incubated with primary and secondary antibodies. The immunoblots were washed with TBST (TBS with 0.1\% Tween-20). Finally, the HRP-conjugated protein was probed with an ECL chemiluminescence kit (Millipore). The signal intensity was calculated with Image Lab.

\section{Co-immunoprecipitation}

Cells in 40-mm dishes were washed carefully with pre-chilled PBS and lysed with $1 \mathrm{~mL}$ of cold IP lysis buffer (Beyotime Biotechnology). After incubation with IP grade primary antibody overnight at $4{ }^{\circ} \mathrm{C}$, Protein A/G Plus-Agarose (Santa Cruz Biotechnology) was added to immunoprecipitate the antigen-antibody complex. Subsequently, the entire complex was washed with pre-chilled IP lysis buffer and boiled with $4 x$ loading buffer. Finally, the supernatant was collected for Western blotting.

\section{Immunofluorescence}

HUVECs were seeded onto cover glasses and allowed to adhere overnight. After stimulation with the indicated treatments, the cells were processed for immunostaining according to the manufacturer's protocol (Beyotime Institute of Biotechnology, Shanghai, China). Briefly, the cells were washed with PBS and then fixed with $4 \%$ paraformaldehyde before permeabilization with $0.2 \%$ Triton X-100. After blocking with goat serum, the cells were incubated with a primary antibody against STAT3 (Proteintech, 1:100 dilution) or CaMKII (Santa Cruz, 1:100 dilution) overnight at $4{ }^{\circ} \mathrm{C}$, followed by incubation with Alexa Fluor 594- or 488-conjugated secondary antibody (1:200 dilution, Thermo Fisher Scientific, Shanghai, China) for $2 \mathrm{~h}$. The cells were then counterstained with DAPI for another 10 min and washed with PBS three times before they were imaged with a confocal microscope (Zeiss LSM 710).

Statistical analysis

The quantitative data are expressed as the mean \pm SEM. Statistical comparisons between groups were performed using one-way ANOVA, followed by Student's $t$-test. A probability value of $P<$ 0.05 was deemed statistically significant.

\section{RESULTS}

Effects of ZYZ-803 on STAT3 and CaMKII phosphorylation ZYZ-803 upregulated STAT3 (Tyr705) and CaMKII (Thr286) phosphorylation in HUVECs on a similar time-course, and both phosphorylation levels peaked at $30 \mathrm{~min}$ (Fig. 1a). In addition, these increases were blocked in the presence of a STAT3 inhibitor (WP1066 $2 \mu \mathrm{M}$ ) as well as a CaMKII inhibitor KN93 (Fig. 1b).
STAT3 and CaMKII are involved in ZYZ-803-induced angiogenesis in HUVECs

Angiogenesis involves several processes, including endothelial cell migration, proliferation, and tube formation. To test directly whether the activities of STAT3 and CaMKII contribute to the angiogenesis induced by ZYZ-803, the effects of WP1066 or KN93 on each of these processes were investigated.

As displayed in Fig. 2, disruption of the STAT3 or CaMKII signaling pathway inhibited multiple aspects of ZYZ-803-induced angiogenesis. ZYZ-803 promoted HUVECs proliferation (Fig. 2a), migration (Fig. 2b, c), and tube formation (Fig. 2d). After treatment with WP1066 or KN93, HUVECs proliferation, migration, and tube formation were significantly inhibited. To further verify the proangiogenic mechanism of ZYZ-803 ex vivo, rat aortic rings were prepared and embedded in Matrigel. The number of outgrowths of branch-like microvessels was increased after ZYZ803 treatment for 6 days (Fig. 2e). As expected, the positive effect of ZYZ-803 was counteracted by WP1066 and KN93. Consistently, exposure to ZYZ-803 also increased the protein expression levels of proliferation markers (cyclin D1 and PCNA) in HUVECs. In addition, treatment with WP1066 and KN93 decreased the ZYZ803-induced cyclin D1 and PCNA expression level increases (Fig. 2f).

STAT3 and CaMKII are involved in ZYZ-803-induced angiogenesis in vivo under hind limb ischemic conditions

To further explore the effects of STAT3 and CaMKII on ZYZ-803induced angiogenesis in vivo, WP1066 (an inhibitor of STAT3) and KN93 (an inhibitor of CaMKII) were injected into mice with HLI. A serial analysis of hind limb perfusion by laser Doppler imaging revealed significantly less flow recovery in both ZYZ-803 + WP1066- and ZYZ-803 + KN93-treated mice than in ZYZ-803treated mice (Fig. 3a). As expected, WP1066 or KN93 administration resulted in a significant decrease in vascular density (Fig. 3b) and damage to the morphological shape of the gastrocnemius muscle (Fig. 3c).

STAT3 knockdown suppresses ZYZ-803-induced HUVECs angiogenesis but upregulates CaMKII expression

Our observations indicated that the phosphorylation levels of STAT3 and CaMKII peaked almost simultaneously after exposure to ZYZ-803 (Fig. 1a). Furthermore, ZYZ-803-induced CaMKII phosphorylation was reversed by WP1066, a selective tyrosinekinase inhibitor of STAT3, whereas the CaMKII inhibitor KN93 did not influence STAT3 activity (Fig. 1b). These results suggest that there may be signal transduction from STAT3 to CaMKII. Based on these results, we transfected STAT3 siRNA into HUVECs to silence STAT3 expression and further confirmed the crucial role of STAT3 in ZYZ-803-induced angiogenesis in vitro. As shown in Fig. 4c, the knockdown of STAT3 significantly decreased cell proliferation, migration, and tube formation, which was in line with the results above (Fig. 2). However, STAT3 siRNA failed to abolish ZYZ-803-induced CaMKII phosphorylation. Indeed, silencing STAT3 expression resulted in increased total CaMKII expression (Fig. 4a).

The interaction between STAT3 and CaMKII was enhanced after ZYZ-803 treatment

To further explore the similar time-course of STAT3 and CaMKII phosphorylation induced by ZYZ-803, we performed a coimmunoprecipitation (Co-IP) assay, which revealed that the interaction between STAT3 and CaMKII was markedly enhanced $30 \mathrm{~min}$ after ZYZ-803 stimulation (Fig. 5a). Additionally, a double fluorescent labeling experiment showed that STAT3 was co-localized with CaMKII after ZYZ-803 treatment (Fig. 5b). 
a
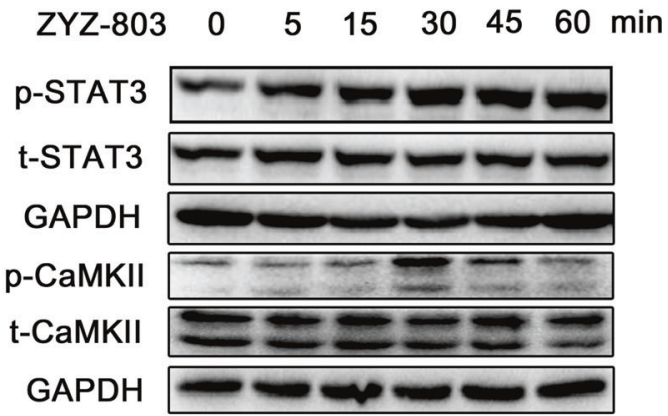

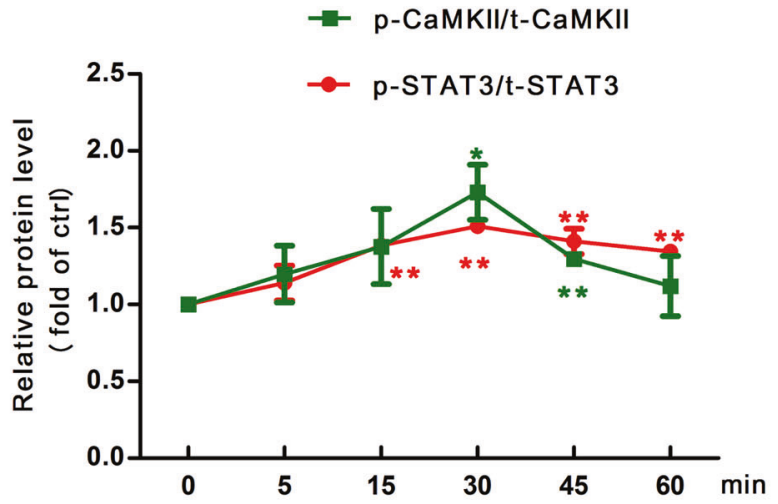

b
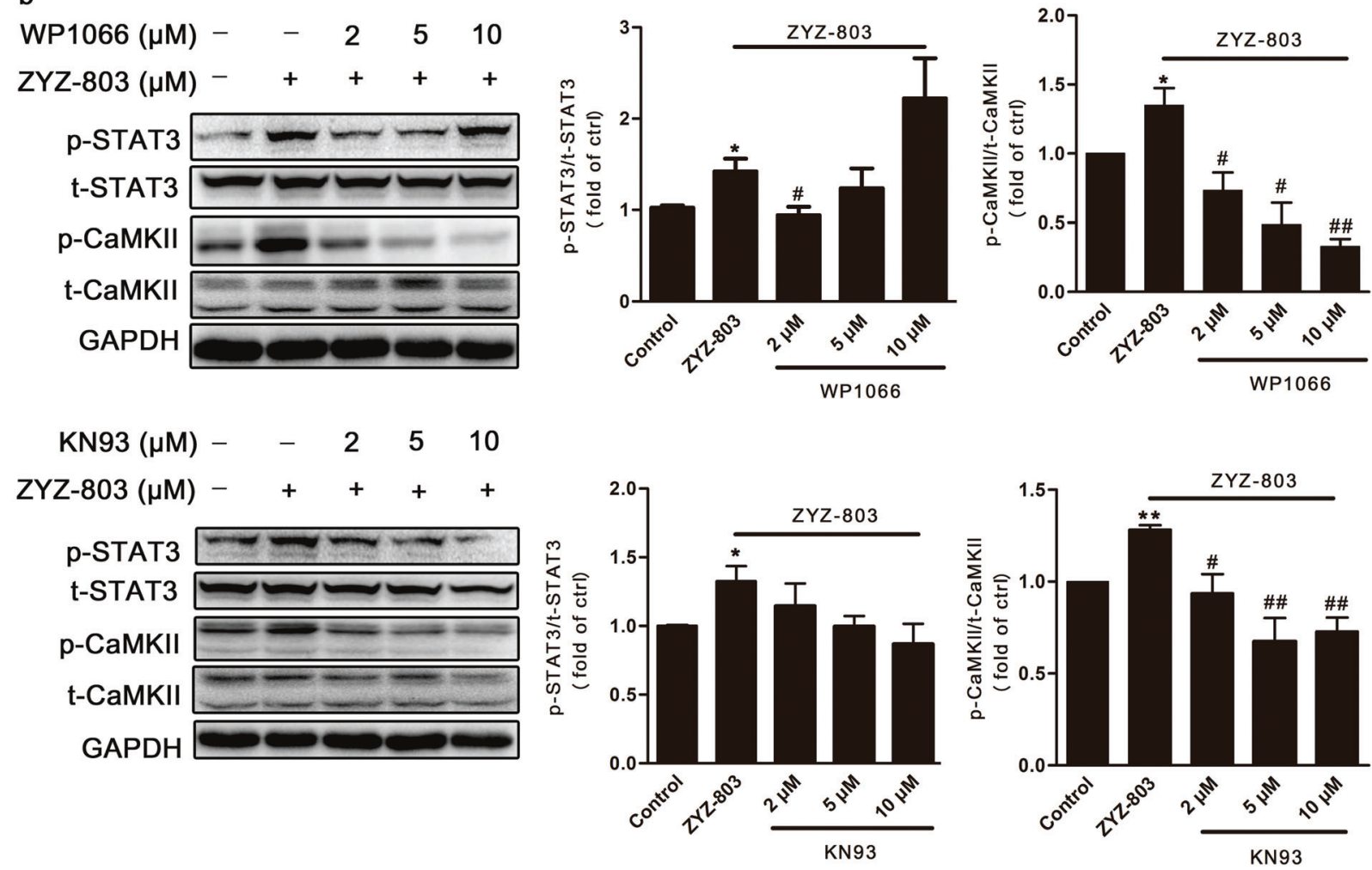

Fig. 1 Effects of ZYZ-803 on STAT3 and CaMKII phosphorylation. a HUVECs were treated with ZYZ-803 (1 $\mu$ M) for different time. b HUVECs were pretreated with WP1066 or KN93 for $1 \mathrm{~h}$, followed by ZYZ-803 $(1 \mu \mathrm{M})$ for $30 \mathrm{~min}$. Protein levels were assessed by Western blot. $n=3$ independent experiments. ${ }^{*} P<0.05,{ }^{*} P<0.01$ versus control; ${ }^{\#} P<0.05,{ }^{\# \#} P<0.01$ versus ZYZ-803 treatment group

ZYZ-803 induced the nuclear translocation of STAT3

To investigate how STAT3 activated downstream molecules, cytoplasmic and nuclear proteins from HUVECs were isolated. We detected an increase in nuclear STAT3 expression, with a mild decrease in cytoplasmic STAT3 levels (Fig. 6a). Corresponding with the Western blot results, significant STAT3 translocation from the cytosol to the nucleus was observed in HUVECs by confocal microscopy after ZYZ-803 treatment (Fig. 6b).

Effects of PAG and L-NAME on STAT3 and CaMKII phosphorylation In our previous work [4], pretreatment with cystathionine $y$-lyase (CSE) and/or endothelial NO synthase (eNOS)-specific siRNAs or inhibitors decreased ZYZ-803-induced angiogenesis in vitro and in vivo, indicating a synergistic interaction of $\mathrm{H}_{2} \mathrm{~S}$ and $\mathrm{NO}$ in the development of angiogenesis. To confirm that ZYZ-803 activates the STAT3/CaMKII pathway in angiogenesis via cooperative $\mathrm{H}_{2} \mathrm{~S}$ NO-mediated mechanisms, the CSE inhibitor DL-propargylglycine (PAG) and the eNOS inhibitor $L-\mathrm{N}^{G}$-nitro arginine methyl ester
(L-NAME) were administered to HUVECs. In the case of STAT3 activation, its phosphorylation level was significantly increased by ZYZ-803 treatment (Fig. 1a). Here, we observed that these effects of ZYZ-803 were attenuated by PAG and/or L-NAME (Fig. 7), Likewise, similar results were found for CaMKII phosphorylation levels, supporting the assumption that ZYZ-803 activates the STAT3/CaMKII pathway in angiogenesis via $\mathrm{H}_{2} \mathrm{~S}$-NO-mediated mechanisms.

\section{DISCUSSION}

Herein, we used a novel hybrid donor of $\mathrm{H}_{2} \mathrm{~S}$ and NO that was previously shown to exhibit significant angiogenic activity both in vitro and in vivo [4]. Our present study identified STAT3 and CaMKII as positive regulators of endothelial cell angiogenesis, which was consistent with previous studies [13, 21]. Accumulating evidence supports cross-talk between STAT3 and CaMKII in other types of cells, including intestinal endothelial cells [18], 


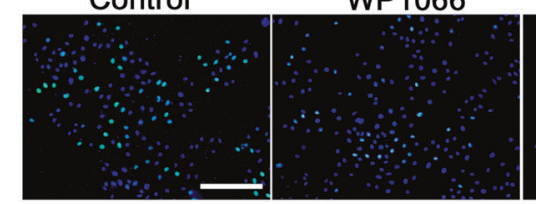

b

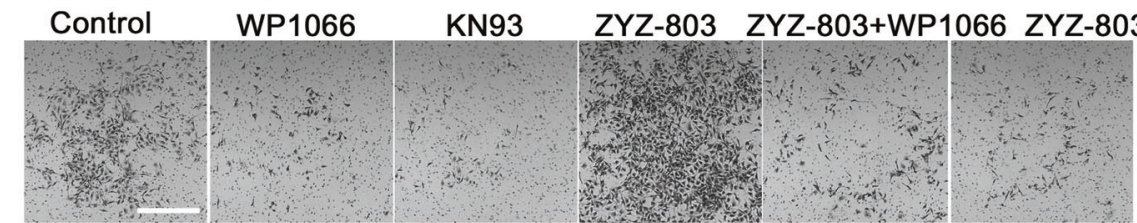

C

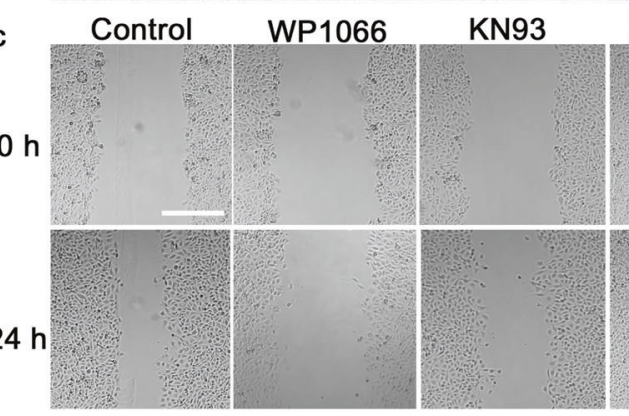

d

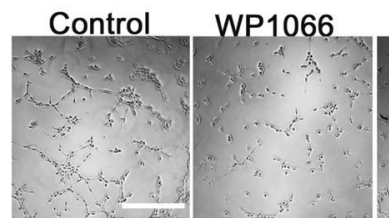

e

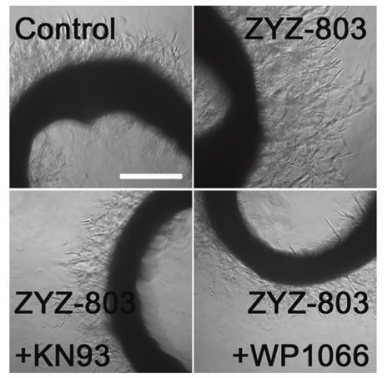

KN93

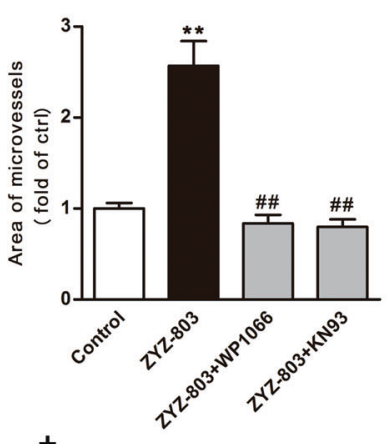

KN93

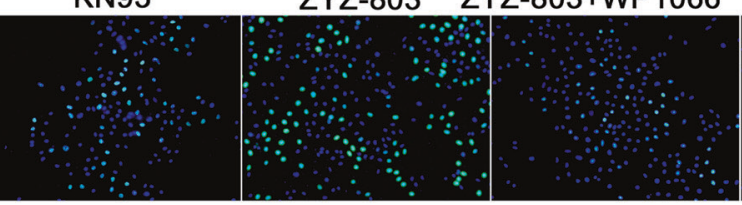

ZYZ-803 ZYZ-803+WP1066

ZYZ-803+KN93

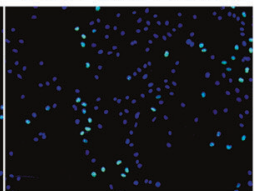

ZYZ-803 ZYZ-803+WP1066 ZYZ-803+KN93
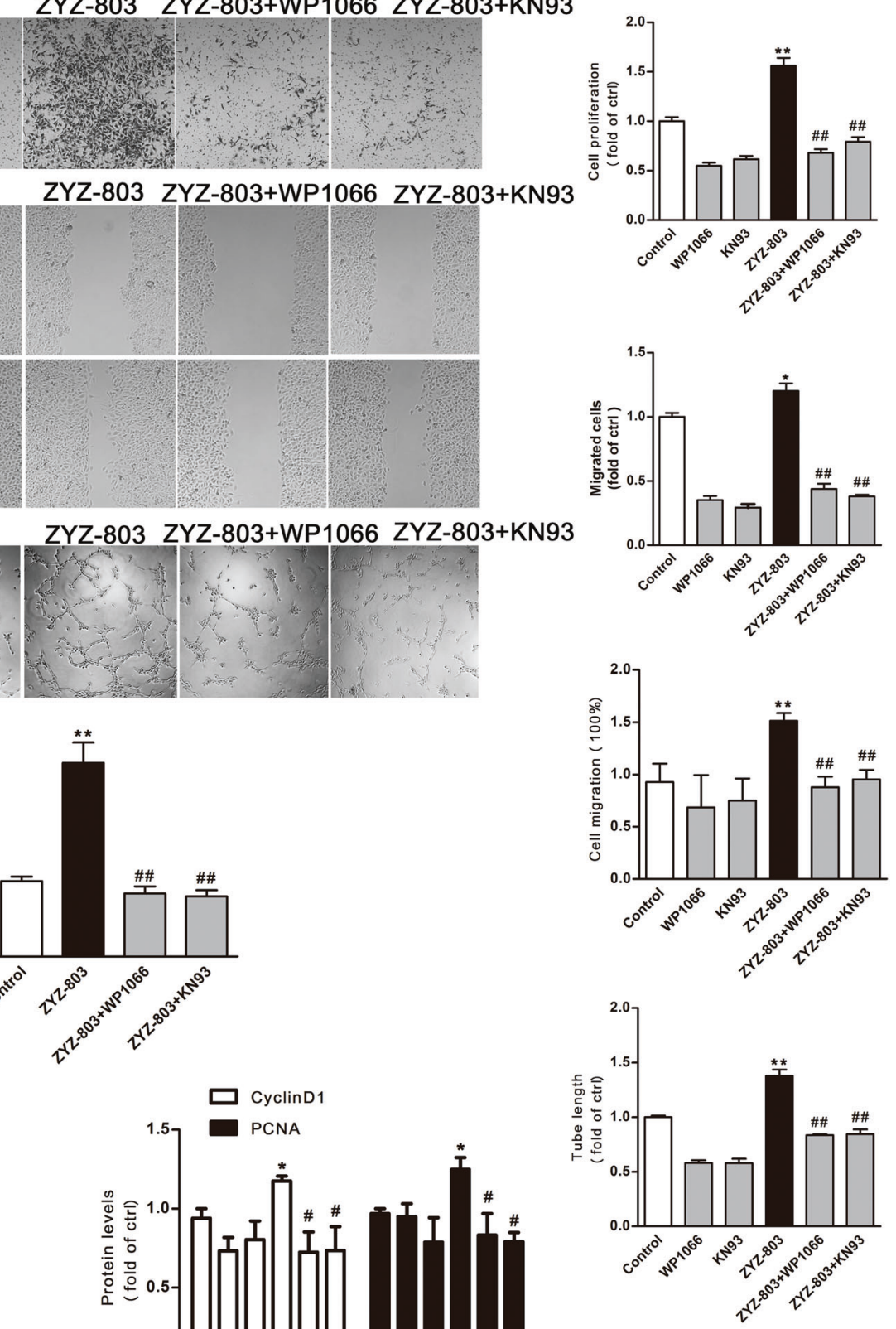

f
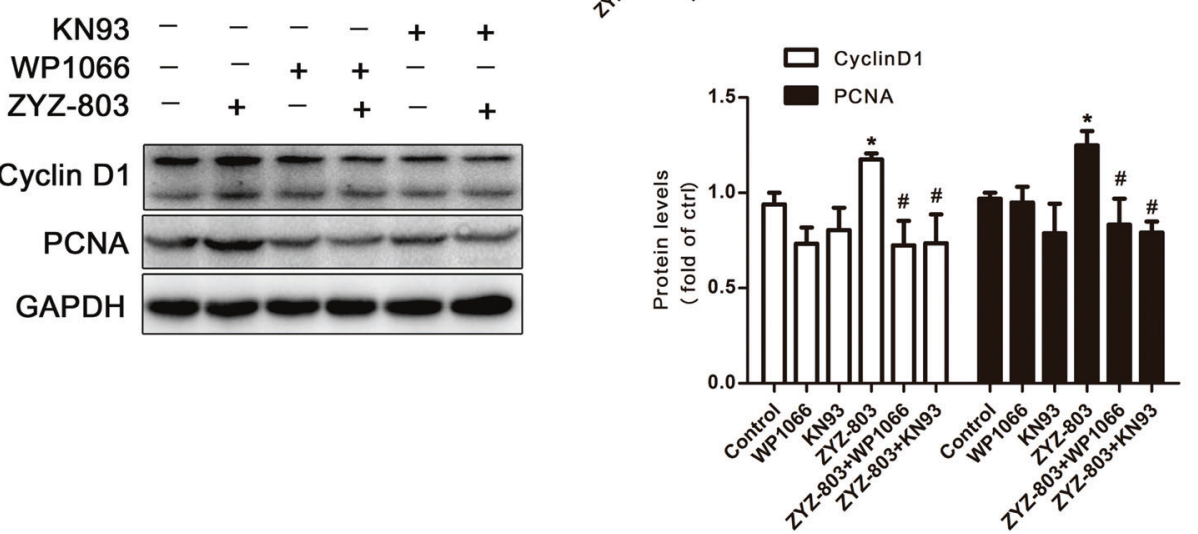
a
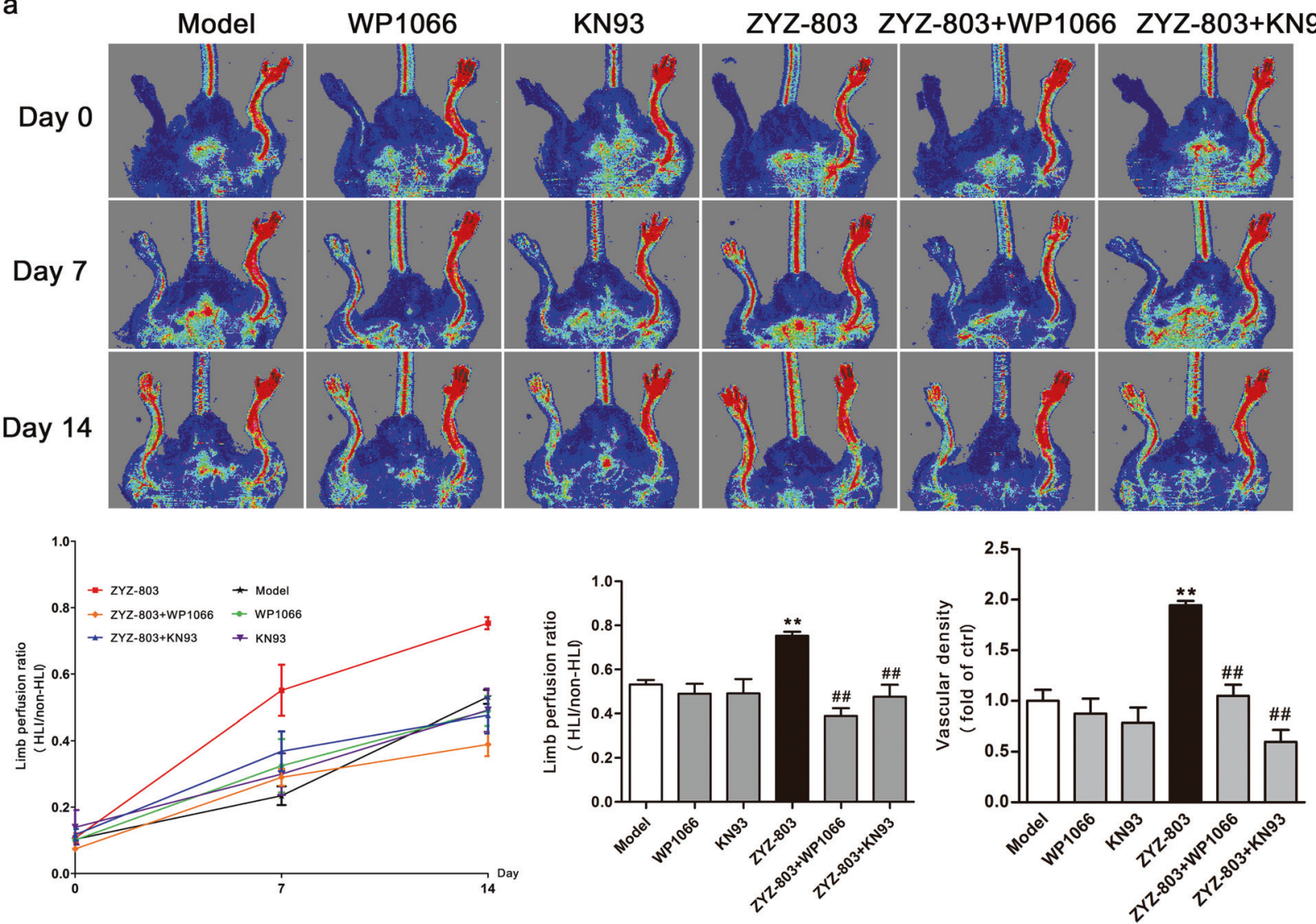

b

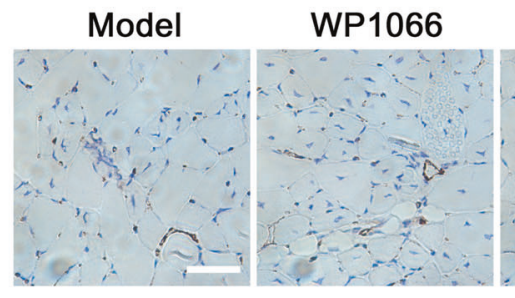

KN93

ZYZ-803 ZYZ-803+WP1066 ZYZ-803+KN93

C

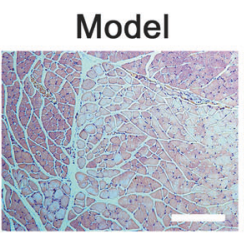

WP1066
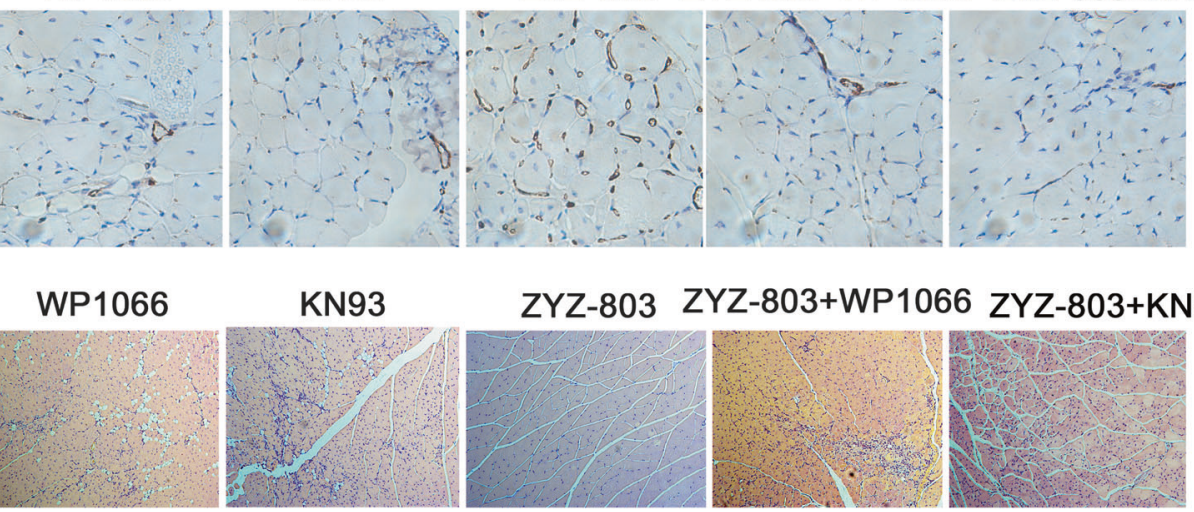

KN93

ZYZ-803

ZYZ-803+WP1066 ZYZ-803+KN93

Fig. 3 STAT3 or CaMKII inhibition prevented ZYZ-803-induced angiogenesis in vivo under ischemic condition. a HLI was surgically induced via femoral artery ligation in 8-week-old C57BL/6J mice. Then the C57BL/6J mice were injected intraperitoneally with ZYZ-803 (8.7 mg/kg per day) or WP1066 $(5.0 \mathrm{mg} / \mathrm{kg}$ per day) or KN93 $(5.0 \mathrm{mg} / \mathrm{kg}$ per day) the day after HLI induction. MoorLDI2-2 laser Doppler imaging system was used to follow the changes in blood flow recovery over time. $n \geq 5$ for each group, ${ }^{* *} P<0.01$ versus model; ${ }^{\# \#} P<0.01$ versus ZYZ-803 treatment group. b Fourteen days after HLI, mice were sacrified, and the gastrocnemius muscles were fixed in paraformaldehyde immediately after they were excised from the operative hind limbs, and then embedded in paraffin. The endothelial cells in the tissue were stained with a rabbit polyclonal CD31 antibody. Scale bar, $20 \mu \mathrm{m}$. c H\&E staining of sections from gastrocnemius muscle of mice 14 days after HLI. Scale bar, $100 \mu \mathrm{m}$

leukemia stem cells [22], myeloid leukemia cells [19], and $\mathrm{H} 9 \mathrm{c} 2$ cells [17]; some of these studies even defined CAMKII as an upstream effector. Likewise, we observed a physical interaction between STAT3 and CaMKII that was markedly enhanced at $30 \mathrm{~min}$, which was exactly the time when the phosphorylation levels of the two proteins peaked after ZYZ803 stimulation.

In Fig. 1b, 2 MM WP1066 (STAT3 inhibitor) successfully blocked the activation of STAT3 induced by ZYZ-803, while $10 \mu \mathrm{M}$ WP1066 did not. Regarding this finding, we assume that the increase in
STAT3 phosphorylation level resulted from non-specific binding in the cells when the dose of WP1066 exceeded a certain concentration. Similar phenomena have been reported and discussed [23]. Many kinase inhibitors have been designed to be structural analogues of ATP, competitively binding to the protein kinase active site to inhibit kinase self-phosphorylation and downstream signal transduction. However, this binding pocket is common to all protein kinases, which means that a particular inhibitor might bind to more than one protein kinase in cells. The compounds are more likely to target many proteins with similar 
a

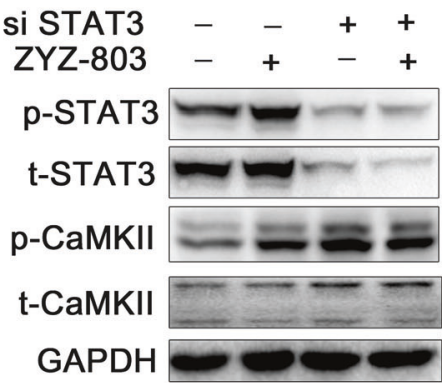

b

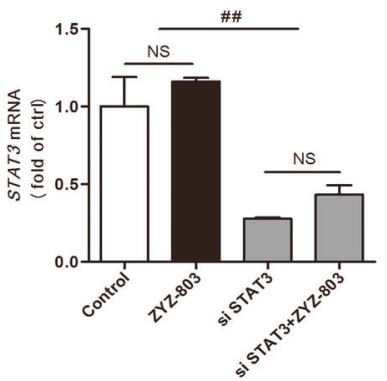

C

ctrl
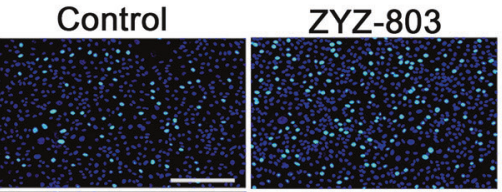

st si
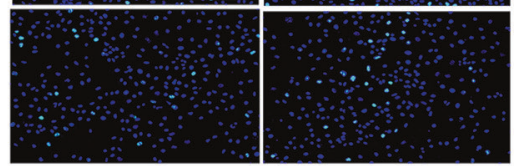

Control

ZYZ-803

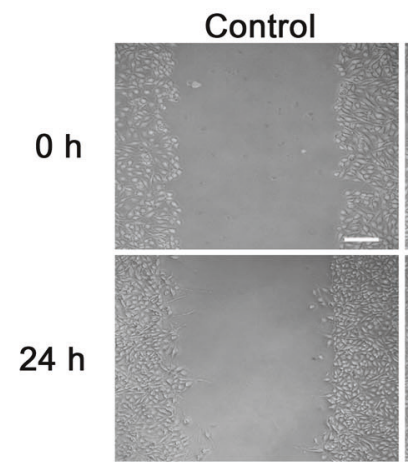

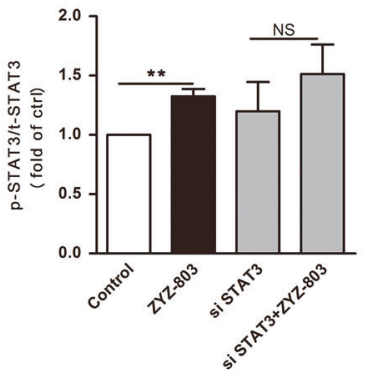
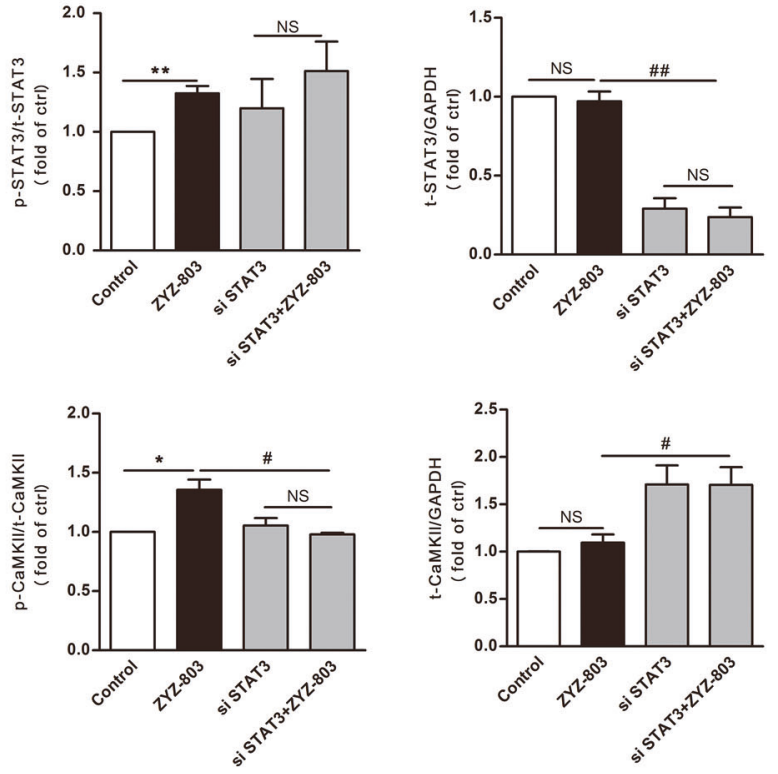
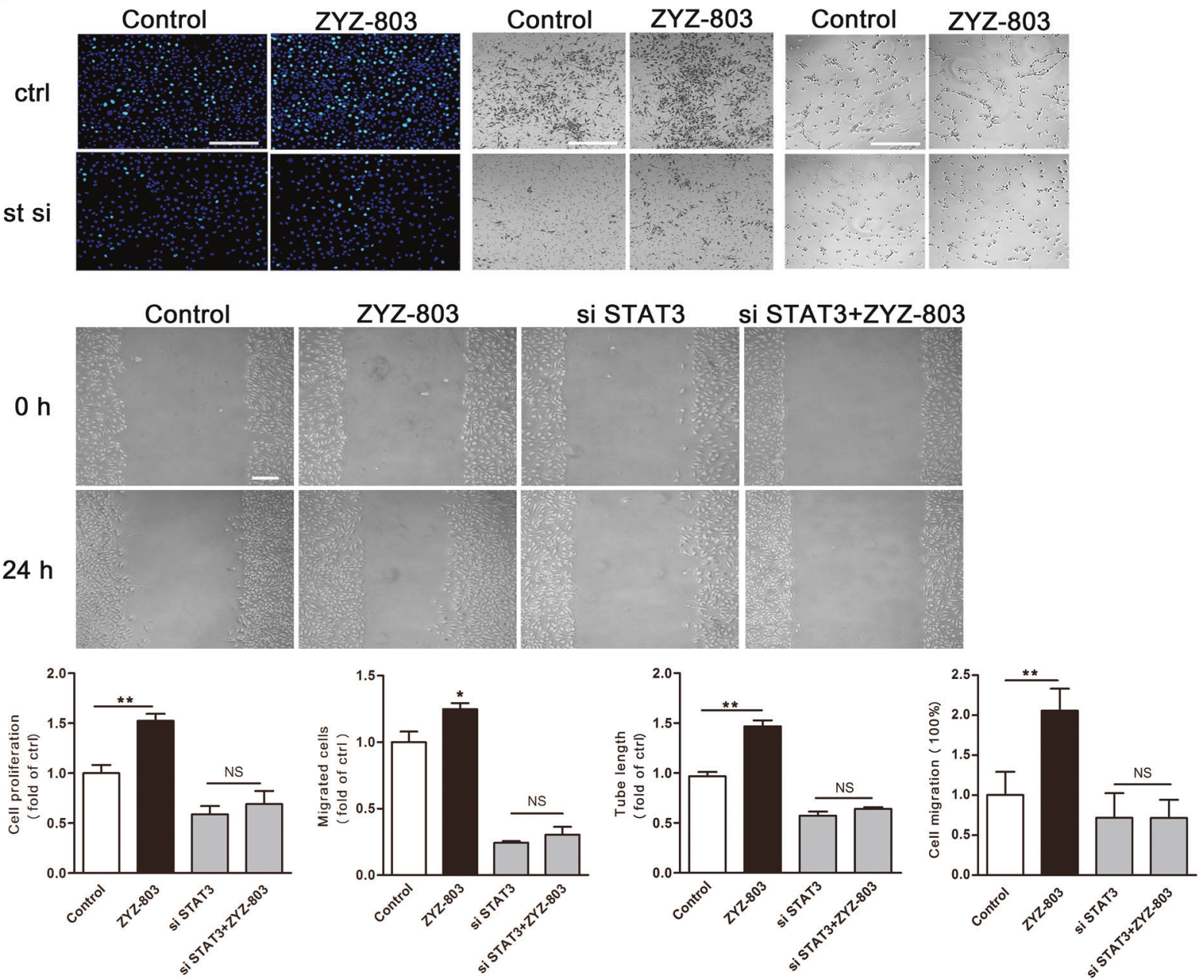

Fig. 4 Effects of STAT3 siRNA on ZYZ-803-induced angiogenesis in HUVECs. a Knockdown of STAT3 inhibited ZYZ-803-induced STAT3 phosphorylation, but upregulated the expression of CaMKII. HUVECs were treated with ZYZ-803 (1 $\mu$ M) for 30 min after STAT3 silencing, which was performed by transfection using an RNAiMAX kit for $48 \mathrm{~h}$. b The mRNA level of STAT3 was quantified using quantitative RT-PCR. HUVECs were treated with ZYZ-803 $(1 \mu \mathrm{M})$ for $30 \mathrm{~min}$ after transfection with siRNA (STAT3) for $48 \mathrm{~h}$. c For proliferation assay, transwell migration assay as well as wound-healing assay, HUVECs were treated with ZYZ-803 $(1 \mu \mathrm{M})$ for $24 \mathrm{~h}$, for tube formation assay, cells were treated with ZYZ-803 $(1 \mu \mathrm{M})$ for $6 \mathrm{~h}$ after transfection with siRNA (STAT3) for $48 \mathrm{~h}$. Scale bars, $100 \mu \mathrm{m} . n=3$ independent experiments. ${ }^{*} P<0.05,{ }^{*} P<0.01$ versus control; ${ }^{\#} P<0.05$, ${ }^{\# \#} P<0.01$ versus $Z Y Z-803$ treatment group 
a

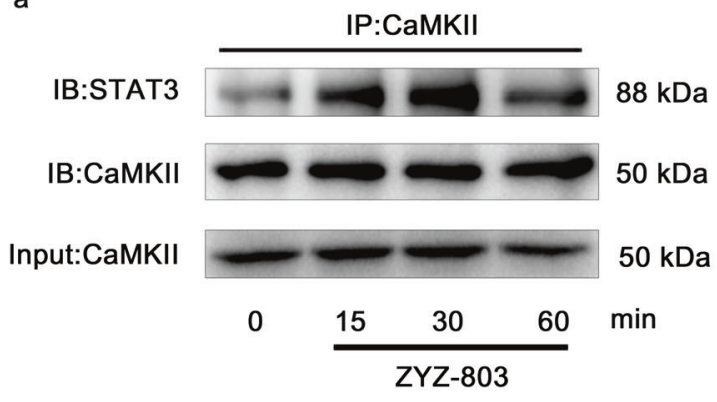

b

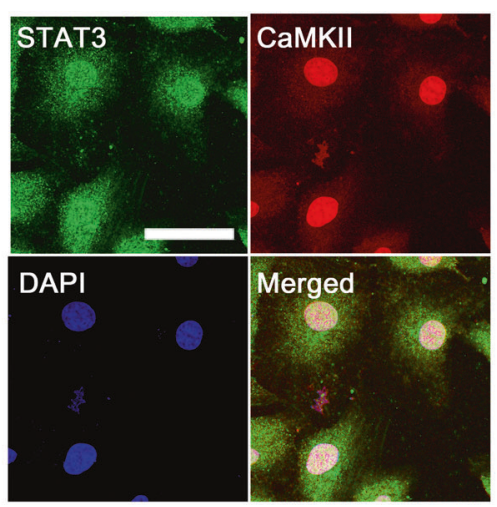

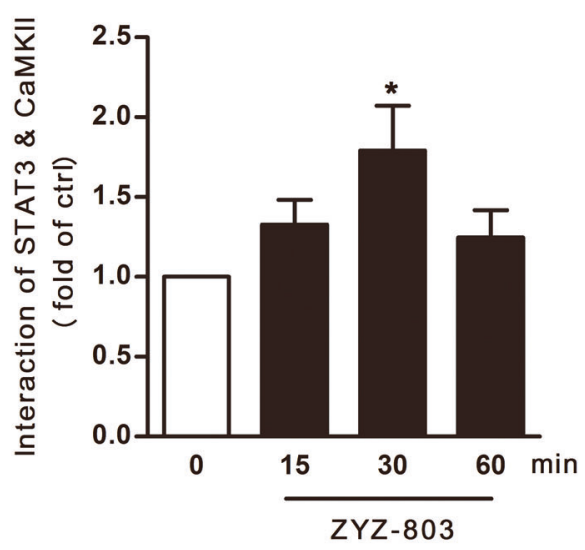

ZYZ-803

Fig. 5 The interaction between STAT3 and CaMKII was enhanced after ZYZ-803 treatment. a STAT3 protein levels in complexes immunoprecipitated with CaMKII antibody. HUVECs were treated with ZYZ-803 (1 $\mu \mathrm{M})$ for 15, 30, 60 min. b HUVECs were fixed and specifically stained with STAT3 antibody (green) and CaMKII antibody (red). DAPI (blue) indicates the nucleus. Scale bar, $10 \mu \mathrm{m}$. $n=3$ independent experiments. ${ }^{*} P<0.05$ versus control

a
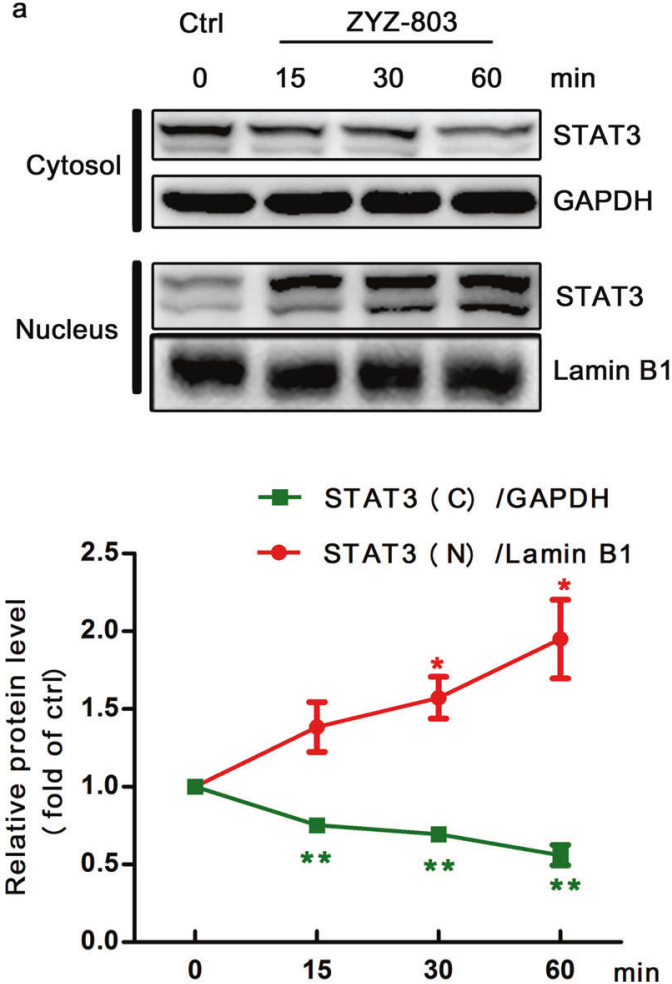

b

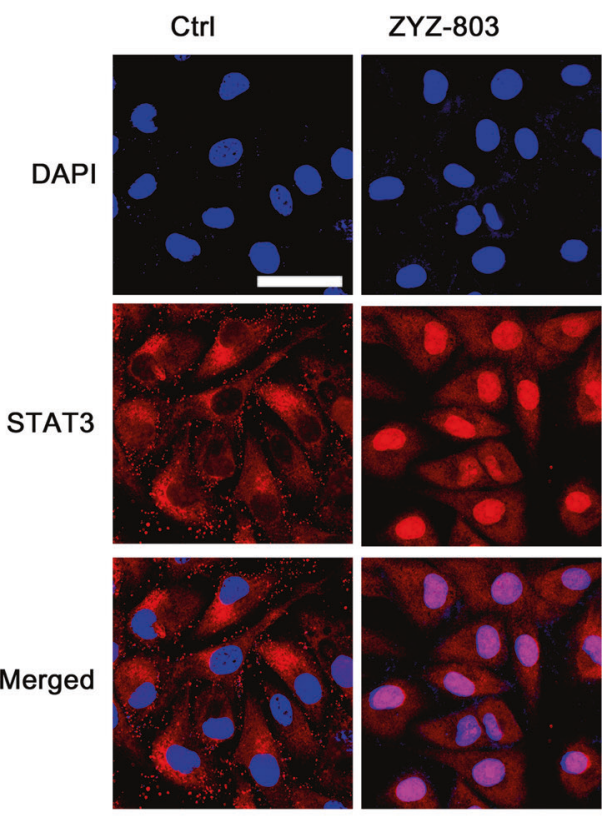

Fig. 6 ZYZ-803 induced the nuclear translocation of STAT3. a HUVECs were treated with ZYZ-803 (1 $\mu$ M) for 15, 30, 60 min, and then, cytoplasmic and nuclear proteins were isolated for Western blotting. GAPDH and Lamin B1 were used as a loading control for cytoplasmic and nuclear proteins, respectively. b HUVECs were treated with ZYZ-803 $(1 \mu \mathrm{M})$ for $30 \mathrm{~min}$ and then stained with specific antibody (red) or DAPI (blue). Scale bar, $10 \mu \mathrm{m} . n=3$ independent experiments. ${ }^{*} P<0.05,{ }^{* *} P<0.01$ versus control 

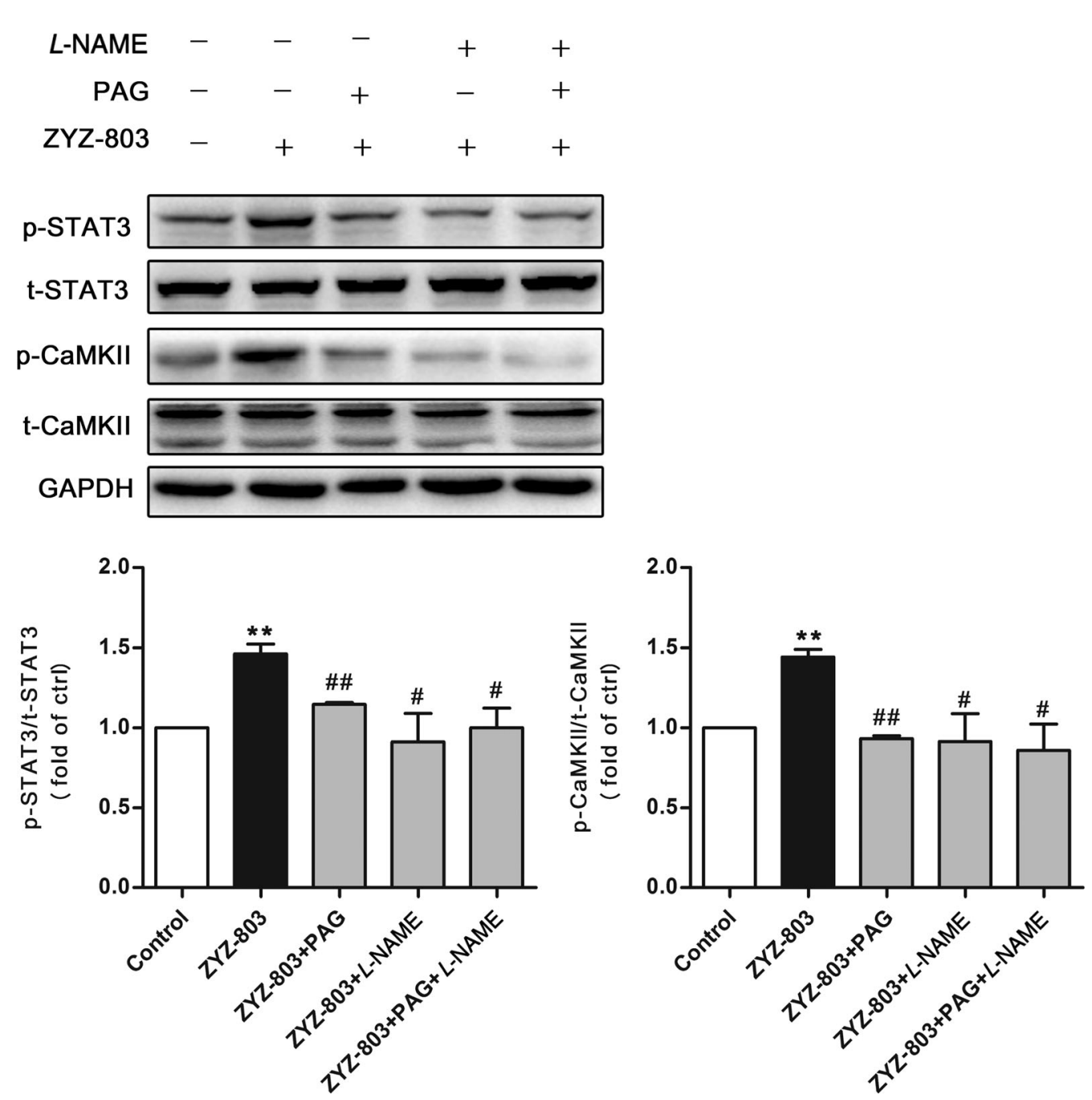

Fig. 7 PAG and L-NAME blocked the phosphorylation of STAT3 and CaMKII induced by ZYZ-803. Cells were pretreated with PAG (5 mM) and/or L-NAME $(5 \mathrm{mM})$ for $1.5 \mathrm{~h}$ followed by ZYZ-803 $(1 \mu \mathrm{M})$ for $30 \mathrm{~min}$. The cell lysates were immunoblotted with p-STAT3, STAT3, p-CaMKII, CaMKII antibodies. $n=3$ independent experiments. ${ }^{*} P<0.01$ versus control; ${ }^{\#} P<0.05,{ }^{\# \#} P<0.01$ versus ZYZ-803 treatment group

conformations, especially for those inhibitors effective in cells at concentrations of only $>10 \mu \mathrm{M}$ [24]. Using the lowest possible concentration of the inhibitor helps to ensure the specificity of the compound. The IC $\mathrm{C}_{50}$ value of WP1066 for B16 cells is $2.43 \mu \mathrm{M}$, and that for HEL cells is $2.3 \mu \mathrm{M}$ as indicated by the manufacturer's information. Of course, to validate this hypothesis, further study should be conducted in the future.

In our current study, blockade of p-STAT3 with WP1066 decreased the phosphorylation of CaMKII within $2 \mathrm{~h}$ without affecting the total protein level (Fig. 1b), but after transfection with siRNA for $48 \mathrm{~h}$, the total protein level of CaMKII increased under the STAT3 deficit (Fig. 4a). These contrasting results may lead to the common question of whether the phenotype has to be congruent between RNAi and small-molecule approaches. The basic mechanisms of kinase inhibition and RNAi need to be understood to answer this question. Specifically, small inhibitors are known to exert their effect by rapid pharmacologic blockade of the protein kinase, while the genetic method of siRNA transfection interferes with the expression of proteins with specific sequences by degrading the complementary mRNA. These two methods involve different mechanisms. Therefore, the phenotypes need not always be consistent [24]. It has been reported that there is a lack of congruence between siRNA and small-molecule inhibitors against p110 $\beta$, a catalytic kinase of the class IA PI(3)K family, as well as Aurora B [24].
Here, we intended to knock down CaMKII expression to further explore the relationship between STAT3 and CaMKII. However, there are at least four isoforms $(\alpha, \beta, \delta$, and $\gamma)$ of CaMKII encoded by different genes [25]. Thus, identifying which isoform is the dominant isoform in endothelial cells will contribute to further understanding the relationship between STAT3 and CaMKII.

Moreover, off-target effects are another problem that cannot be ignored in the use of siRNAs as a gene knockdown tool [26]. Studies have revealed that short interfering RNAs can trigger unexpected effects on the levels of non-specific proteins, but these off-target effects are generally invisible unless the genes related to pivotal cellular responses are investigated thoroughly [27]. Thus, we have no evidence that the activation of CaMKIIassociated pathways induced by siRNA (STAT3) accounts for these unexpected effects. New methods for detecting non-specific effects induced by siRNA may help us to determine this issue.

The results above indicated that there might be cross-talk between the STAT3- and CaMKII-related pathways, which may have synergistic effects on the angiogenesis induced by ZYZ-803, although other distinct STAT3/CAMKII-dependent mechanisms remain elusive. Thus, it is worth focusing our future research on how these two key factors communicate with each other under ZYZ-803 stimulation in HUVECs.

It has been previously demonstrated that the nuclear translocation of STAT3 is essential for endothelial cell migration and tube 


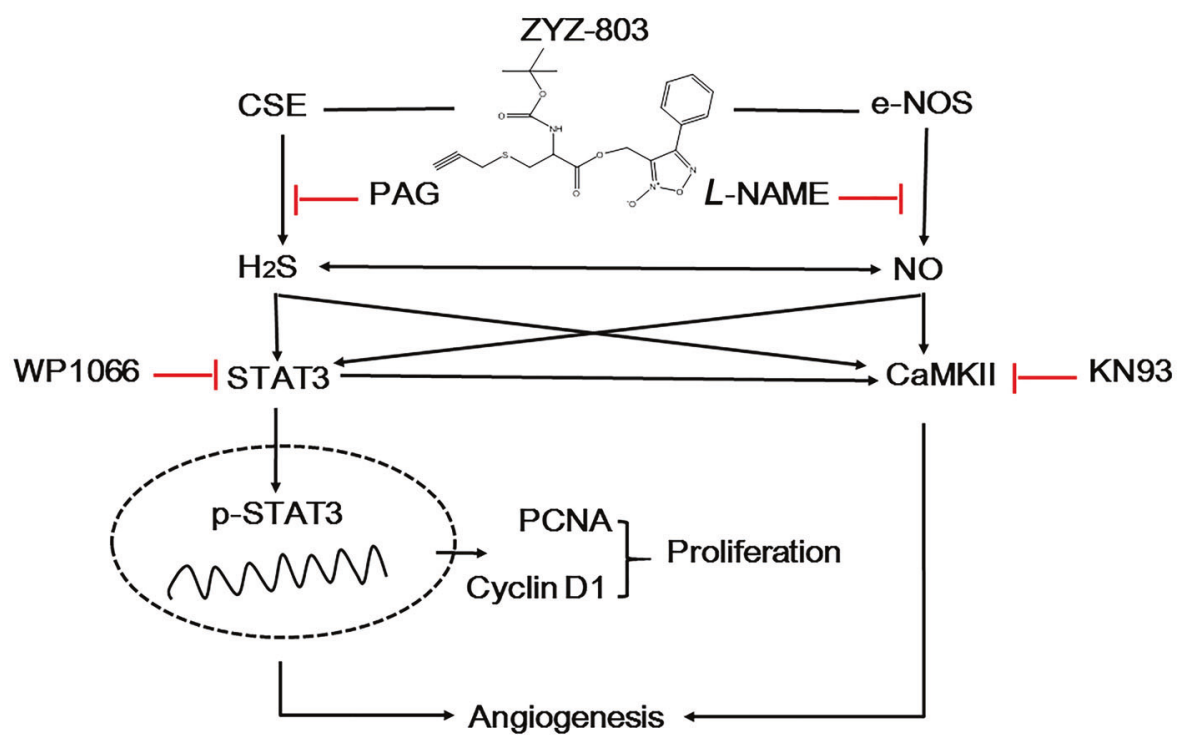

Fig. 8 Hypothetical mechanistic model of STAT3/CaMKII signaling modulation by ZYZ-803 in angiogenesis

formation [28]. In this study, we also observed nuclear translocation of phosphorylated STAT3 following ZYZ-803 stimulation (Fig. 6) and then STAT3-associated CaMKII activation. However, the detailed mechanism as well as the downstream pathway remain to be further explored.

In our recently published work, $\mathrm{H}_{2} \mathrm{~S}$ and $\mathrm{NO}$ generated from ZYZ-803 were mutually required for the physiological regulation of endothelial homeostasis [4]. These two gasotransmitters cooperatively regulated vascular tone [2] and attenuated left ventricular remodeling and dysfunction during the development of heart failure [3]. Here, we also confirmed that ZYZ-803 activates the STAT3/CaMKII pathway in angiogenesis via $\mathrm{H}_{2} \mathrm{~S}-\mathrm{NO}$-mediated mechanisms.

Several studies have reported that the $\mathrm{H}_{2} \mathrm{~S}$-STAT3 axis and the NO-CaMKII axis are associated with angiogenesis. In this study, we observed cross-talk between these two signal pathways. As shown in Fig. 7, PAG (CSE inhibitor) attenuated not only the activation of STAT3 but also the activation of CaMKII. This interesting phenomenon indicated that the activation of both STAT3 and CaMKII was regulated by $\mathrm{H}_{2} \mathrm{~S}$ generated from ZYZ-803. Likewise, L-NAME attenuated the activation of CaMKII as well as STAT3, which indicated that the activation of both STAT3 and CaMKII was also regulated by NO. Thus, $\mathrm{H}_{2} \mathrm{~S}$ and $\mathrm{NO}$ generation from $\mathrm{ZYZ}-803$ work synergistically to regulate the activation of STAT3 and CaMKII in angiogenesis (Fig. 8).

In conclusion, our present study has provided compelling evidence that both STAT3 and CaMKII function as positive regulators of ZYZ-803-induced endothelial angiogenesis in vivo and in vitro, which further sheds light on the beneficial role of ZYZ-803 in STAT3/CaMKII-related cardiovascular diseases.

\section{ACKNOWLEDGEMENTS}

This work was supported by grants from the National Natural Science Foundation of China (Nos. 81402956, 81573421, and 81330080), the Shanghai Committee of Science and Technology of China (Nos. 16431902000 and 19QA1401500), and the Shanghai Chenguang Program (No. 14CG03).

\section{AUTHOR CONTRIBUTIONS}

$Y Z Z, Y C M$, and $Y X$ designed the research; $Y X, T D$, and RZ performed the research; LLC and $B T$ provided technology support; $Y Z Z, Y C M$, and $Y X$ analyzed the data and wrote the paper.

\section{ADDITIONAL INFORMATION}

Conflict of interest: The authors declare no competing interests.

\section{REFERENCES}

1. Sivakumar B, Harry LE, Paleolog EM. Modulating angiogenesis: more vs less. JAMA. 2004;292:972-7.

2. Wu D, Hu Q, Ma F, Zhu YZ. Vasorelaxant effect of a new hydrogen sulfide-nitric oxide conjugated donor in isolated rat aortic rings through cGMP pathway. Oxid Med Cell Longev. 2016;2016:1-10.

3. Wu D, Hu Q, Xiong Y, Zhu D, Mao Y, Zhu YZ. Novel $\mathrm{H}_{2} \mathrm{~S}-\mathrm{NO}$ hybrid molecule (ZYZ803) promoted synergistic effects against heart failure. Redox Biol. 2018;15:243-52.

4. Hu Q, Wu D, Ma F, Yang SN, Tan B, Xin H, et al. Novel angiogenic activity and molecular mechanisms of ZYZ-803, a slow-releasing hydrogen sulfide-nitric oxide hybrid molecule. Antioxid Redox Signal. 2016;25:498-514.

5. Hayashida R, Kondo K, Morita S, Unno K, Shintani S, Shimizu Y, et al. Diallyl trisulfide augments ischemia-induced angiogenesis via an endothelial nitric oxide synthase-dependent mechanism. Circ J. 2017;81:870-8.

6. Saha S, Chakraborty PK, Xiong X, Dwivedi SKD, Mustafi SB, Leigh NR, et al Cystathionine $\beta$-synthase regulates endothelial function via protein $S$-sulfhydration. FASEB J. 2016;30:441-56.

7. Kimura $\mathrm{H}$. Hydrogen sulfide and polysulfides as signaling molecules. Proc Jpn Acad Ser B Phys Biol Sci. 2015;91:131-59.

8. Lee CZ, Xue Z, Hao Q, Yang G, Young WL. Nitric oxide in vascular endothelial growth factor-induced focal angiogenesis and matrix metalloproteinase- 9 activity in the mouse brain. Stroke. 2009;40:2879-81.

9. Lu A, Wang L, Qian L. The role of eNOS in the migration and proliferation of bonemarrow derived endothelial progenitor cells and in vitro angiogenesis. Cell Biol Int. 2015;39:484-90.

10. Zhang $H$, Chen JC, Sheibani L, Lechuga TJ, Chen D. Pregnancy augments VEGFstimulated in vitro angiogenesis and vasodilator ( $\mathrm{NO}$ and $\mathrm{H}_{2} \mathrm{~S}$ ) production in human uterine artery endothelial cells. J Clin Endocrinol Metab. 2017;102: 2382-93.

11. Kanagy NL, Szabo C, Papapetropoulos A. Vascular biology of hydrogen sulfide. Am J Physiol-Cell Physiol. 2017;312:C537-49.

12. Kan JT, Guo W, Huang CR, Bao GZ, Zhu YC, Zhu YZ. S-propargyl-cysteine, a novel water-soluble modulator of endogenous hydrogen sulfide, promotes angiogenesis through activation of signal transducer and activator of transcription 3 . Antioxid Redox Signal. 2014;20:2303-16.

13. Banumathi E, O'Connor A, Gurunathan S, Simpson DA, McGeown JG, Curtis TM VEGF-induced retinal angiogenic signaling is critically dependent on $\mathrm{Ca}^{2+} \mathrm{sig}$ naling by $\mathrm{Ca}^{2+} /$ calmodulin-dependent protein kinase II. Invest Ophthalmol Vis Sci. 2011;52:3103-11.

14. Faehling $M$, Kroll J, Föhr KJ, Fellbrich G, Mary U, Trischler G, et al. Essential role of calcium in vascular endothelial growth factor A-induced signaling: mechanism of the antiangiogenic effect of carboxyamidotriazole. FASEB J. 2002; 16:1805-7. 
15. Schneider JC, El Kebir D, Chéreau C, Lanone S, Huang XL, De Buys Roessingh AS,

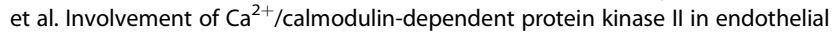
NO production and endothelium-depended relaxation. Am J Physiol Heart Circ Physiol. 2003;284:H2311-9.

16. Wu Y, He M, Ye J, Ma S, Huang W, Wei $Y$, et al. Activation of ATP-sensitive potassium channels facilitates the function of human endothelial colony-forming cells via $\mathrm{Ca}^{2+} / \mathrm{Akt} / \mathrm{eNOS}$ pathway. J Cell Mol Med. 2017;21:609-20.

17. Zhao L, Cheng G, Jin R, Afzal MR, Samanta A, Xuan Y, et al. Deletion of interleukin6 attenuates pressure overload-induced left ventricular hypertrophy and dysfunction. Circ Res. 2016;118:1918-29.

18. Ma X, Meng Z, Jin L, Xiao Z, Wang X, Tsark WM, et al. CAMK2 $\gamma$ in intestinal epithelial cells modulates colitis-associated colorectal carcinogenesis via enhancing STAT3 activation. Oncogene. 2017;36:4060-71.

19. Si J, Collins SJ. Activated $\mathrm{Ca}^{2+} /$ calmodulin-dependent protein kinase llgamma is a critical regulator of myeloid leukemia cell proliferation. Cancer Res. 2008;68:3733-42.

20. Yu J, Dardik A. A murine model of hind limb ischemia to study angiogenesis and arteriogenesis. Methods Mol Biol. 2018;1717:135-43.

21. Bartoli M, Platt D, Lemtalsi T, Gu XL, Brooks SE, Marrero MB, et al. VEGF differentially activates STAT3 in microvascular endothelial cells. FASEB J. 2003;17:1562-4.
22. Gu Y, Chen T, Meng Z, Gan Y, Xu X, Lou G, et al. CaMKII, a critical regulator of CML stem/progenitor cells, is a target of the natural product berbamine. Blood. 2012;120:4829-39.

23. Langendorf CG, Scott JW, Kemp BE. Fake inhibitors: AMPK activation trumps inhibition. Cell Chem Biol. 2017;24:775-7.

24. Weiss WA, Taylor SS, Shokat KM. Recognizing and exploiting differences between RNAi and small-molecule inhibitors. Nat Chem Biol. 2007;3:739-44.

25. Cai H, Liu D, Garcia JG. CaM Kinase II-dependent pathophysiological signalling in endothelial cells. Cardiovasc Res. 2007;77:30-4.

26. Khan AA, Betel D, Miller ML, Sander C, Leslie CS, Marks DS. Transfection of small RNAs globally perturbs gene regulation by endogenous microRNAs. Nat Biotechnol. 2009;27:549-55.

27. Scacheri PC, Rozenblatt-Rosen O, Caplen NJ, Wolfsberg TG, Umayam L, Lee JC, et al. Short interfering RNAs can induce unexpected and divergent changes in the levels of untargeted proteins in mammalian cells. Proc Natl Acad Sci USA. 2004;101:1892-7.

28. Yahata Y, Shirakata $Y$, Tokumaru S, Yamasaki K, Sayama K, Hanakawa $Y$, et al. Nuclear translocation of phosphorylated STAT3 is essential for vascular endothelial growth factor-induced human dermal microvascular endothelial cell migration and tube formation. J Biol Chem. 2003;278:40026-31. 